\title{
2-Oxoquinoline Arylaminothiazole Derivatives in Identifying Novel Potential Anticancer Agents by Applying 3D-QSAR, Docking, and Molecular Dynamics Simulation Studies
}

\author{
Reda El-Mernissi ${ }^{1}$, Khalil El Khatabi ${ }^{1}$, Ayoub Khaldan ${ }^{1}$, Larbi ElMchichi ${ }^{1}$, Md Shahinozzaman ${ }^{2}$, \\ Mohammed Aziz Ajana ${ }^{1, *}$, Tahar Lakhlifi ${ }^{1}$, Mohammed Bouachrine ${ }^{1,3,4}$ \\ ${ }^{1}$ Molecular chemistry and Natural Substances Laboratory, Faculty of Science, University Moulay Ismail, \\ Meknes, Morocco. \\ ${ }^{2}$ Department of Nutrition and Food Science, University of Maryland, College Park, MD 20742, USA. \\ ${ }^{3}$ EST Khenifra, Sultan Moulay Sliman University, Beni mellal, Morocco. \\ ${ }^{4}$ Department of General Chemistry, Polytechnic University of Bucharest, 1 Polizu st.
}

*Corresponding author: Mohammed Aziz Ajana, email: a.ajanamohammed@fs.umi.ac.ma

Received May $7^{\text {th }}, 2021$; Accepted November $30^{\text {th }}, 2021$.

DOI: http://dx.doi.org/10.29356/jmcs.v66i1.1578

\begin{abstract}
Tubulin plays an indispensable role in regulating various important cellular processes. Recently, it is known as a hopeful therapeutic target for the rapid division of cancer cells. Novel series of 2-oxoquinoline arylaminothiazole derivatives have been recently identified as promising tubulin inhibitors with potent cytotoxicity activity against HeLa cancer cell line. In this study, a 3D-QSAR approach by using CoMFA and CoMSIA techniques was applied to the reported derivatives to understand their pharmacological essentiality contributing to the tubulin inhibition activity and selectivity. The optimum CoMFA and CoMSIA models were found to have significant statistical reliability and high predictive ability after internal and external validation. By analyzing the contour maps, the electrostatic and hydrophobic interactions were found to be crucial for improving the inhibitory activity and four novel tubulin inhibitors (Compounds D1, D2, D3, and D4) were designed based on the validated 3D-QSAR models. Moreover, the docking findings showed that residues Gln136, Val238, Thr239, Asn167, Val 318 and Ala 316 played important roles for quinoline binding to tubulin. Among the newly designed compounds, compound D1 with the highest total scoring was subjected to detailed molecular dynamics (MD) simulation and compared to the most active compound. The conformational stability of compound $\mathbf{D} 1$ complexed with tubulin protein was confirmed by a 50 -ns molecular dynamics simulation, which was congruent with molecular docking.
\end{abstract}

Keywords: 3D-QSAR; molecular docking; MD simulation; quinoline; cancer.

Resumen. La tubulina juega un papel indispensable en la regulación de varios procesos celulares importantes. Recientemente, se le ha reconicodo como un agente promisorio para atacar la rápida división de las células cancerosas. Últimamente se ha identificado una nueva serie de derivados de arilaminotiazo-2-oxoquinolina como potenciales inhibidores de la tubulina, con una elevada actividad citotóxica contra la línea celular de cáncer HeLa. En este estudio, se aplicó a los derivados informados un estudio 3D-QSAR mediante el uso de técnicas CoMFA y CoMSIA para comprender los factores farmacológicos que contribuyen a la actividad como inhibidor y selectivo de la tubulina. Se encontró que los modelos CoMFA y CoMSIA óptimos tienen una confiabilidad estadística significativa y una alta capacidad predictiva después de la validación interna y externa. $\mathrm{Al}$ analizar los mapas de contorno, se descubrió que las interacciones electrostáticas e hidrófobas eran cruciales para mejorar la actividad inhibidora y se diseñaron cuatro nuevos inhibidores de la tubulina (compuestos D1, 
D2, D3 y D4) basados en los modelos 3D-QSAR validados. Además, los hallazgos de acoplamiento mostraron que los residuos Gln136, Val238, Thr239, Asn167, Val 318 y Ala 316 desempeñaron papeles importantes en la unión de la quinolina a la tubulina. Entre los compuestos de nuevo diseño, el compuesto D1 con la puntuación total más alta se sometió a una simulación detallada de dinámica molecular (MD) y se comparó con el compuesto más activo. La estabilidad conformacional del compuesto D1 unido a la proteína tubulina se confirmó mediante una simulación de dinámica molecular de $50 \mathrm{~ns}$, que fue congruente con el acoplamiento molecular.

Palabras clave: QSAR-3D, Docking molecular, Simulaciones de Dinámica Molecular, quuinolina, cáncer.

\section{Introduction}

Cancer is a complicated, pervasive, and fatal illness. It is recognized as a significant public health issue worldwide [1]. It starts as cells become abnormal and begin to expand outside of their normal borders, and it can then infiltrate neighboring areas and spread to other organs of the body [2]. Microtubules, as essential cytoskeleton components, are formed by heterodimers of $\alpha, \beta$-tubulin [3]. They play a key role in a variety of cellular functions, including formation of cell signaling motility regulation, cell shape, intracellular transport and secretion [4,5]. Microtubules interacting drugs have been recognized as promising targets for cancer chemotherapy due to their importance in mitosis and cell division, in which they are known as tubulin dimer cages that play a role in chromosome dynamics, which stops cell division in mitosis [6-8]. Taxanes, vincaalkaloids and colchicine have been identified as cancer chemotherapeutic agents targeting the microtubule cytoskeleton [9]. The discovery of tubulin inhibitors that bind to the active site of colchicine was a key move in the development of antimitotic drugs [10]. Compared to taxanes and vinca alkaloids, colchicine has more therapeutic advantages and better clinical benefits [11].

Quinoline fused heterocycles have been shown to have potent cancer inhibitory efficacy in a wide variety of human cancer cell lines by inducing cell cycle arrest and apoptosis, as well as inhibiting angiogenesis, disrupting cell proliferation, functioning as growth inhibitors, and inhibiting tubulin polymerization [12]. Quinoline, one of the privileged structures in medicinal chemistry, is a well-known heterocyclic compound frequently found in functional organic materials and medicinal drugs. Also, it can be found in natural alkaloids that display substantial and wide-spectrum biological activities like antidiabetic, antiviral, antimalarial, antiinflammatory, antioxidant, antimicrobial and anticancer [13-17]. Due to its biological properties and synthetic usefulness, 2-oxoquinoline, a class of heterocyclic molecular scaffold, has attracted a lot of attention among the different types of active quinoline skeletons.

Furthermore, there has been positive progress in testing tubulin inhibitors in preclinical and clinical trials. Nevertheless, the lower affinity necessitates the development of very efficient tubulin inhibitors, and it is critical to use in silico approaches to design novel tubulin inhibitors having higher activity in order to advance drug research. Here, a 3D-QSAR analysis was conducted on 25 tubulin inhibitors sharing the 2-oxoquinoline scaffold, based on ligand-based alignment. Upon which, rationally novel designed compounds were developed. Carefully molecular docking studies were also carried out to display ligand-protein interactions and calculate of the binding affinity. Based on extra-precision molecular dynamics simulation, the stability of molecularprotein complex system was further investigated. The outcome of this study could be helpful and supportive for the design of novel 2-oxoquinoline arylaminothiazole-based tubulin inhibitors 


\section{Experimental}

\section{Materials and Methods \\ Database}

2-oxoquinoline arylaminothiazole derivatives involved in this work were retrieved from available literature [18] and were used in the development of the 3D-QSAR models. The molecular biological activities of these compounds expressed as IC50 values spanned a range from $(4.4-73.2 \mu \mathrm{M})$ and were converted into the $\mathrm{pIC}_{50}$ (e.g., $\mathrm{pIC}_{50}=-\log \mathrm{IC}_{50}$ ). The whole data was randomly divided into a training set including 20 compounds for the generation of the 3D-QSAR model and an external test set containing 5 compounds for validation (asterisk labeled in Table S1). Table 1 displays the structures of molecules as well as their experimental activity values. The chemical structures of the compounds studied are shown in Fig. 1.

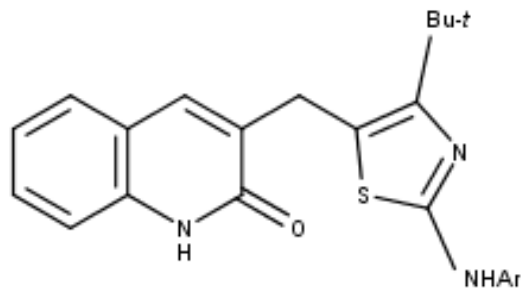

Fig. 1. Structural template of studied compounds.

Table 1. $\mathrm{pIC}_{50}$ values of reported 2-oxoquinoline arylaminothiazole derivatives against tubulin.

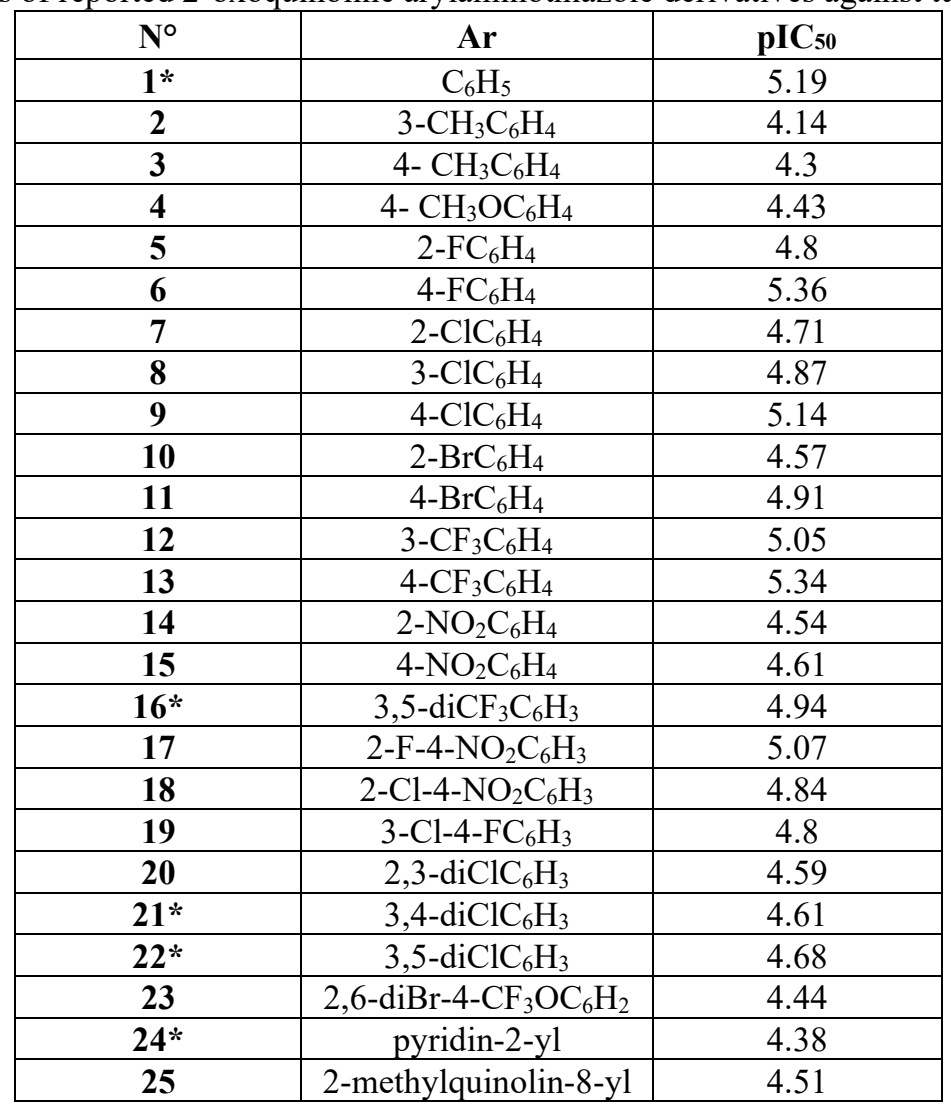

* Test molecule 


\section{Minimization and optimization}

The chemical structures of the reported derivatives were drawn by the builder panel and optimized by using SYBYL-X 2.0 program [19]. Tripos force filled with Gasteiger-Huckel charges with a convergence criterion of $0.01 \mathrm{kcal} / \mathrm{mol}$ were used to optimize the geometry of these compounds [20,21].

\section{Molecular alignment}

One of the most critical steps in deriving stable and reliable 3D-QSAR models is 3D structural alignment. The simple alignment method was used to align the compounds [22], in which the most active compound 6 was served as a structural template for the alignment of the remaining compounds in the data set. The minimized structures were employed as the primal conformations for 3D-QSAR analyses. Fig. 2 depicts the superimposed structures of aligned compounds.

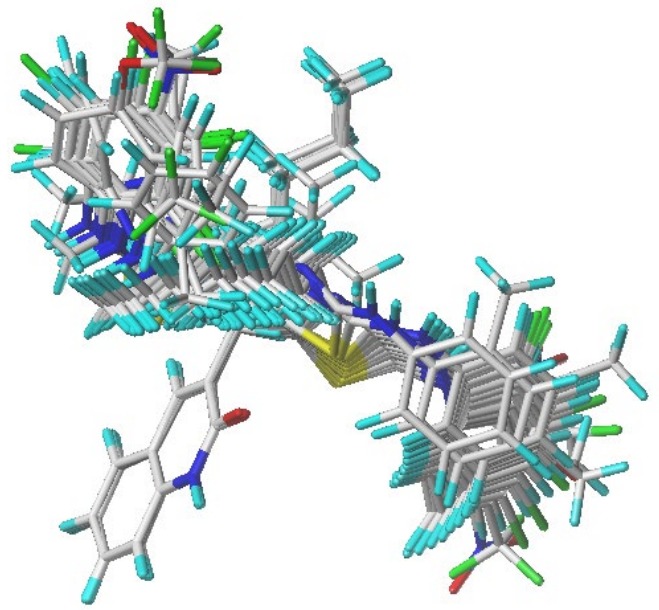

Fig. 2. Aligned molecules by using compound 6 as a structural template.

\section{Development of 3D-QSAR models}

The most common and accurate methods for investigating the relationship between 3D structures and biological activities of compounds were CoMFA and CoMSIA, which were used in the development of the 3DQSAR model [23,24]. CoMFA was developed to quantitatively relate the biological activity changes with the steric and electrostatic characteristics of molecules, whereas CoMSIA might be able to relate the biological activity with steric, electrostatic, hydrogen bond acceptor (HBA), hydrogen bond donor (HBD), and hydrophobic characteristics of molecules. Finally, partial least square analysis was conducted with the value of column filtering set to $2.0 \mathrm{kcal} / \mathrm{mol}$ and the energy cutoff set to $30 \mathrm{kcal} / \mathrm{mol}$ [25], while other parameters were set as default. The 3D-QSAR modeling analysis was carried out by SYBYL-X 2.0 program in standard settings, which ran on Windows 10 operating system.

\section{Partial Least Square (PLS) analysis}

PLS analysis method was performed to describe the linear relationship between the calculated CoMFA and CoMSIA descriptors fields with the reported biological activities [26]. The optimum number of components $(\mathrm{N})$ and the coefficient of cross-validation correlation $\left(\mathrm{Q}^{2}\right)$ were calculated by leave-one-out (LOO) method as the first stage. Based on the previously acquired $\mathrm{N}$ value, a non-cross validation procedure was carried out as the second stage, in which the correlation coefficient (R2), the standard error of estimate (SEE) and F-test value (F) were calculated. the regression fitting of developed 3D-QSAR models was described based on the high values of $\mathrm{Q}^{2}$ and $\mathrm{R}^{2}\left(\mathrm{Q}^{2}>0.50\right.$ and $\left.\mathrm{R}^{2}>0.60\right)$ [27,28], with a low standard error estimation (SEE) value which helps to choose the optimal model. A reliable QSAR model with more accurate predictability must also provide an optimal number of components. External validation was used to assess the reliability of the optimal models 
using the test set compounds, since it is the most valuable validation method, which requires that the appropriate condition (rext2 $>0.6$ ) must be fulfilled [29].

\section{Y-randomization test}

The Y-randomization technique is a commonly used approach to assess the possibility of chance correlation in constructed models [30]. The dependent variables ( $\mathrm{pIC}_{50}$ values) were ascribed at random, and new QSAR models were developed with the initial independent variables. This process is replicated several times. We considered that the optimal models were not influenced by any chance correlation if the QSAR models were obtained by shuffling the $\mathrm{pIC}_{50}$ values gave low $\mathrm{R}^{2}$ and $\mathrm{Q}^{2}$ values than the nonrandomized models, which indicates the reliability of these models.

\section{Molecular docking}

Molecular docking is widely employed using Surflex-Dock module in Sybyl 2.0 for further screening of query compounds and illustrating their potential interaction modes of activity with protein targets. The crystal structure 3E22 was given by the RSCB Protein Data Bank. Both water molecules and the initial ligand were isolated from the protein using Discovery Studio 2016 software [31], and the binding modes were visualized using PyMOL software [32]. In this study, flexible molecular docking was applied in the docking process. The ligand was treated as fully flexible, while the receptor was kept rigid. The docking findings were then analyzed in depth to determine whether the higher predicted activities and docking scores of the selected compounds were appropriate. A compound with higher docking score among the newly designed compounds was further selected for molecular dynamics simulation and compared to the most active compound of the studied data set.

\section{Molecular Dynamics (MD) simulation}

To investigate the dynamic conformational changes of the selected docked compounds with tubulin protein, a MD simulation was done by Desmond Dynamics software [33] employing OPLS force field [34]. The initial structures used for MD simulations were the protein-ligand complexes obtained by molecular docking. The Leap module was utilized to interpret and extract structural snapshots of the molecular protein topology files. The Particle Mesh Ewald approach was used to calculate long-range electrostatic interactions [35]. The NVT stage was done with a $100 \mathrm{ps}$ MD run to equilibrate the system at a steady temperature of 300 $\mathrm{K}$ by using the Berendsen algorithm. The water molecules and appropriate counter ions $\left(\mathrm{Cl}^{-}\right.$or Na$\left.{ }^{+}\right)$were added for neutralization at $300 \mathrm{~K}$ temperature. By using the steepest descent algorithm (5000 cycles) to execute energy minimization of the solvent system, unwanted contacts between heavy atoms were dislodged. For the overall simulations, the cut-off radius and time stage values were set to 0.8 and $1.25 \mathrm{fs}$, respectively. Finally, a production MD conducted for $50 \mathrm{~ns}$ was performed on each equilibrated system and the trajectory of the simulated system was calculated for every $100 \mathrm{ps}$. The root-mean-square fluctuation (RMSF), root-mean-square deviation (RMSD), gyration radius (Rg) and solvent accessible surface area (SASA) were recorded for $50 \mathrm{~ns}$.

\section{Results and discussion}

\section{D-QSAR analysis}

Internal and external validation were relevant parameters for assessing the accuracy of 3D-QSAR model. The statistical results of 3D-QSAR PLS analysis are outlined in Table 2. The observed and predicted $\mathrm{pIC}_{50}$ of molecules are listed in Table 3. For internal validation, the CoMFA model shows a satisfactory $\mathrm{Q}^{2}$ value of 0.692 for the training set with $\mathrm{N}$ of 5 , an outstanding $\mathrm{R}^{2}$ of 0.875 , F-test of 101.72 and lowest SEE of 0.065 , implying that the model that has been developed is reliable. The steric field contributed $45 \%$ of the total contribution, while the electrostatic field contributed $55 \%$, indicating that the steric field was more significant. The result of CoMSIA model gave an acceptable $\mathrm{Q}^{2}$ of 0.761 with an optimized component of 5 , an excellent $\mathrm{R}^{2}$ of 0.956 , a F-test value of 53.16, and SEE value of 0.09 This indicated that the generated model had a high level of internal predictability. The proportions of steric, electrostatic, HBA, HBD and hydrophobic sites in this model were, respectively, $7 \%, 37 \%, 11 \%, 19 \%$, and $26 \%$. The electrostatic and hydrophobic fields all play 
key roles in the corresponding model, according to the results. The electrostatic field was the most significant interaction between the ligand and the protein target of these three fields. CoMFA and CoMSIA provided outstanding predictive ability for external validation, with $\mathrm{r}_{\mathrm{ext}}{ }^{2}$ values of 0.98 and 0.97 , respectively. This indicated that the external predictability of both models did up to the standard, which demonstrates the robustness of the developed models. The scatter plots of the predicted and observed $\mathrm{pIC}_{50}$ for the data set compounds exhibit that all points are distributed close to the straight line as shown in Fig. 3 . The above findings indicate that the derived models were of good quality.

Table 2. PLS statistics parameters.

\begin{tabular}{|c|c|c|c|c|c|c|c|c|c|c|c|}
\hline Model & \multirow{2}{*}{$\mathbf{Q}^{\mathbf{2}}$} & \multirow{2}{*}{$\mathbf{R}^{\mathbf{2}}$} & \multirow{2}{*}{ SEE } & \multirow{2}{*}{$\mathbf{F}$} & \multirow{2}{*}{$\mathbf{N}$} & $\mathbf{r}_{\text {ext }}{ }^{2}$ & \multicolumn{5}{|c|}{ Fractions } \\
\cline { 7 - 12 } & & & & & & & Ster & Elec & Acc & Don & Hyd \\
\hline CoMFA & 0.692 & 0.875 & 0.065 & 101.72 & 5 & 0.98 & 0.45 & 0.55 & - & - & - \\
\hline CoMSIA & 0.761 & 0.956 & 0.09 & 53.16 & 5 & 0.97 & 0.07 & 0.37 & 0.11 & 0.19 & 0.26 \\
\hline
\end{tabular}

Table 3. Observed and predicted $\mathrm{pIC}_{50}$ of 25 2-oxoquinoline arylaminothiazole derivatives.

\begin{tabular}{|c|c|c|c|c|c|}
\hline \multirow[b]{2}{*}{$\mathbf{N}^{\circ}$} & \multirow[b]{2}{*}{$\begin{array}{c}\text { Observed } \\
\text { pIC }_{50}\end{array}$} & \multicolumn{2}{|c|}{ CoMFA } & \multicolumn{2}{|c|}{ CoMSIA } \\
\hline & & predicted & Residuals & predicted & Residuals \\
\hline $1 *$ & 5.19 & 5.17 & 0.02 & 5.16 & 0.03 \\
\hline 2 & 4.14 & 4.17 & -0.04 & 4.18 & -0.04 \\
\hline 3 & 4.3 & 4.4 & -0.09 & 4.37 & -0.07 \\
\hline 4 & 4.43 & 4.48 & -0.06 & 4.38 & 0.05 \\
\hline 5 & 4.8 & 4.76 & 0.05 & 4.77 & 0.03 \\
\hline 6 & 5.36 & 5.47 & -0.12 & 5.44 & -0.08 \\
\hline 7 & 4.71 & 4.9 & -0.19 & 4.88 & -0.17 \\
\hline 8 & 4.87 & 5.06 & -0.18 & 5 & -0.13 \\
\hline 9 & 5.14 & 5.15 & -0.02 & 5.1 & 0.04 \\
\hline 10 & 4.57 & 4.72 & -0.15 & 4.75 & -0.18 \\
\hline 11 & 4.91 & 5 & -0.08 & 5.01 & -0.1 \\
\hline 12 & 5.05 & 5.22 & -0.17 & 5.12 & -0.07 \\
\hline 13 & 5.34 & 5.45 & -0.12 & 5.35 & -0.01 \\
\hline 14 & 4.54 & 4.58 & -0.04 & 4.56 & -0.02 \\
\hline 15 & 4.61 & 4.69 & -0.08 & 4.67 & -0.06 \\
\hline $16^{*}$ & 4.94 & 5 & -0.05 & 5 & -0.06 \\
\hline 17 & 5.07 & 5.02 & 0.05 & 5.04 & 0.03 \\
\hline 18 & 4.84 & 5 & -0.17 & 4.9 & -0.06 \\
\hline 19 & 4.8 & 4.83 & -0.04 & 4.88 & -0.08 \\
\hline 20 & 4.59 & 4.64 & -0.06 & 4.6 & -0.01 \\
\hline $21 *$ & 4.61 & 4.74 & -0.12 & 4.67 & -0.06 \\
\hline $22 *$ & 4.68 & 4.7 & -0.01 & 4.72 & -0.04 \\
\hline 23 & 4.44 & 4.51 & -0.07 & 4.49 & -0.05 \\
\hline $24 *$ & 4.38 & 4.45 & -0.07 & 4.44 & -0.06 \\
\hline 25 & 4.51 & 4.54 & -0.03 & 4.52 & -0.01 \\
\hline
\end{tabular}



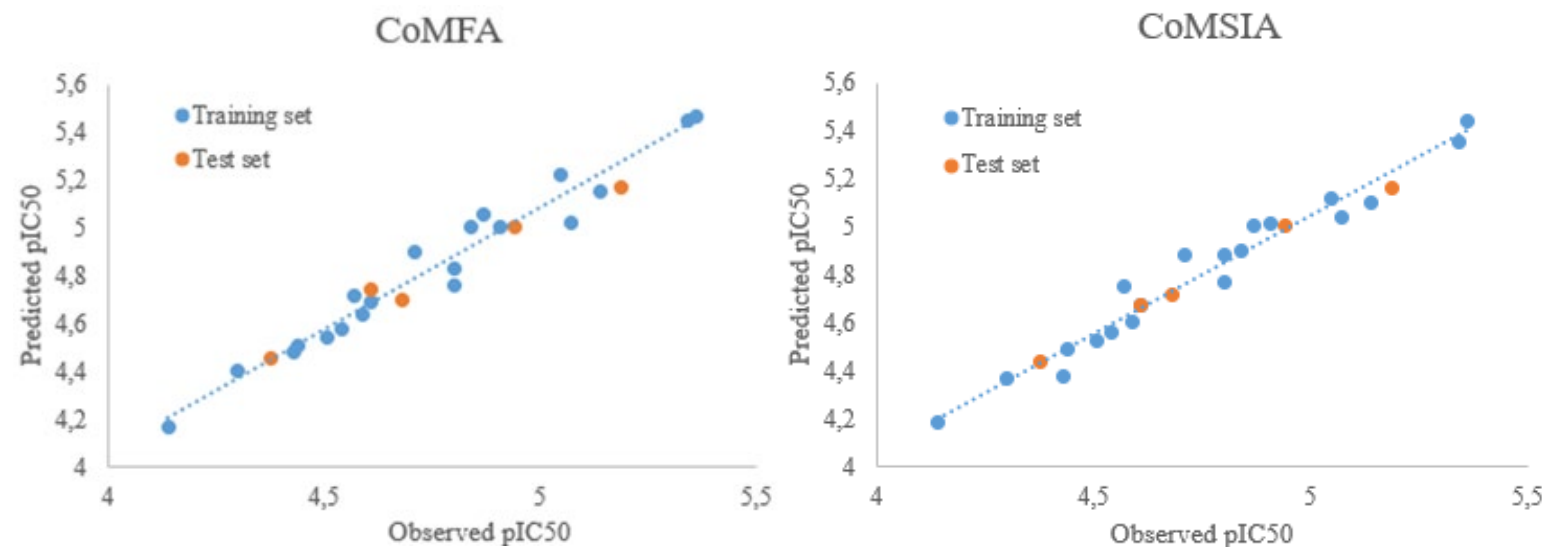

Fig. 3. Plots of observed and predicted $\mathrm{pIC}_{50}$ for CoMFA and CoMSI.

\section{Contour map analysis}

The contour maps were provided to determine the main areas where structural changes (modifying/removing) in CoMFA and CoMSIA fields would affect the inhibitory activity. As a reference for the explanation, compound $\mathbf{6}$ was overlaid on the contours.

\section{CoMFA contour maps}

As the steric field depicted in Fig. 4(a), the green region ( $80 \%$ contribution) represents a steric contribution to potency, while region of yellow ( $20 \%$ contribution) reduces the potency. As shown in the map, a large region of green contour is seen at the 4,5 and 6 positions of the phenyl of aminothiazole, suggesting a preference for less bulky groups. Furthermore, three small sized yellow regions are located at the second and third positions of the same phenyl, which indicates that raising the volume at these positions would not be beneficial to activity. This finding can be explained by noting that compound 10, with bromo-substituted group, is less active than compounds $\mathbf{6}$ and $\mathbf{8}$, with chlor- and fluor-substituted groups, respectively.

Fig. 4(b) shows the electrostatic contour map, with blue areas ( $80 \%$ contribution) indicating the positive electrostatic charge potential related to improved activity and the red areas (20\% contribution) indicating negative charge potential. As shown in the map, a moderately sized red contour is located at the 4 position of the phenyl moiety, indicating that the improvement of protein-binding affinity can be done by enhancing electronegativity in this area. Hence, the $\mathrm{pIC}_{50}$ values of compounds with increased $\pi$-electron density on the 4 position of the phenyl were much higher than that of compounds with an alkyl-substituted group at the same position. Additionally, a blue region near the 2 and 3 positions of the phenyl moiety demonstrates that substituents with positive charges may be beneficial in these regions.
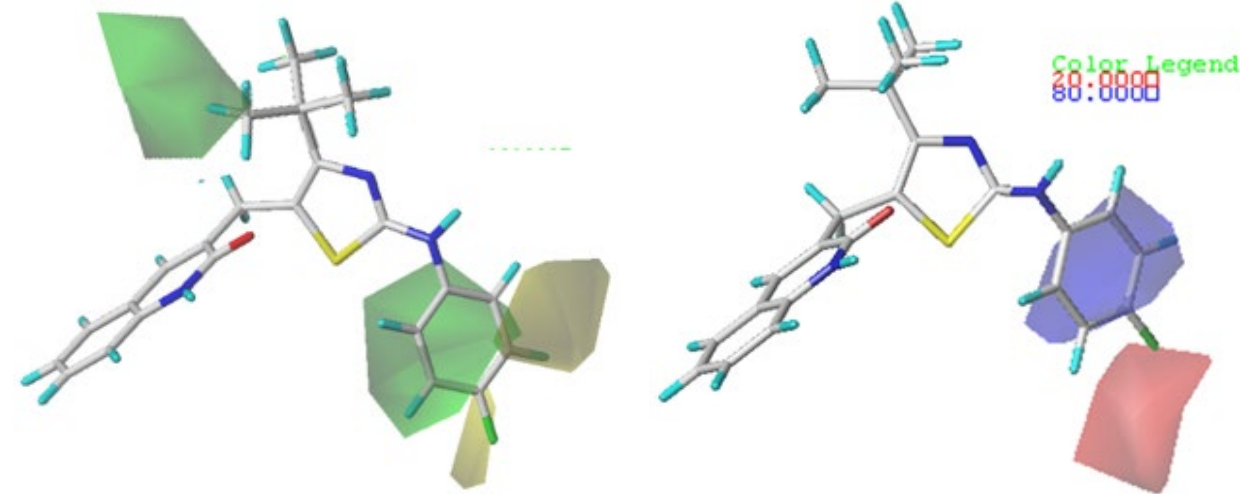

Fig. 4. Contour maps of CoMFA analysis for compound 6. (a) Steric field; (b) Electrostatic field. 


\section{CoMSIA contour map}

In CoMSIA, other parameters such as HBD, HBA and hydrophobic are also obtained, in addition to steric and electrostatic fields as shown in Fig. 5(a), 5(b), and 5(c), respectively. The contours obtained from steric and electrostatic fields were similar to those of the CoMFA model. Accordingly, only the H-bonding and hydrophobic contour maps were further analyzed.

In the HBD field (Fig. 5(a)), the cyan (80\% contribution) color denotes the HBD groups that are favored for the potency, while the purple ( $20 \%$ contribution) contour designates the HBD groups, which are unfavorable for improving the potency. A small region of cyan contour near the $-\mathrm{NH}$ group on the bridge between the thiazole and phenyl ring of compound 6 signifies that the existence of $\mathrm{H}$-bond donor groups leads to improved activity. In the HBA field (Fig. 5(b)), magenta ( $80 \%$ contribution) and red (20\% contribution) colored contours represent the favored and disfavored regions respectively for HBA groups. The magenta contour around the nitro group of the thiazole showed that substituting a H-bond acceptor in this position could improve activity. Moreover, a moderately sized red contour is seen at the first position of the butyl-linked thiazole, indicating that adding HBD groups at this position is reasonable for a positive effect in activity.

Fig. 5(c) shows the hydrophobic contour map, in which the yellow areas ( $80 \%$ contribution) represent hydrophobic potential associated with increased activity, while the white areas ( $20 \%$ contribution) indicate hydrophilic potential. The yellow areas at the 4 and 5 positions on the phenyl ring suggest that hydrophobicity is favored on the overall activity in these regions. For instance, compound $\mathbf{1 3}$ having the 4-trifluoromethyl substituent on the aminothiazole phenyl ring leads to higher activity than due to the presence of the corresponding 4-methyl substituent does in compound 3. This finding is also supported by the higher $\mathrm{pIC}_{50}$ of compounds 9, 11, 13 and 15 than that of compound 4 with an methoxy-substituted group at the position 4 of the same phenyl ring. A region including white contour is located near the position 2 of the aminothiazole phenyl ring, signifying that the presence of wettable substituents in this area may increase the activity.

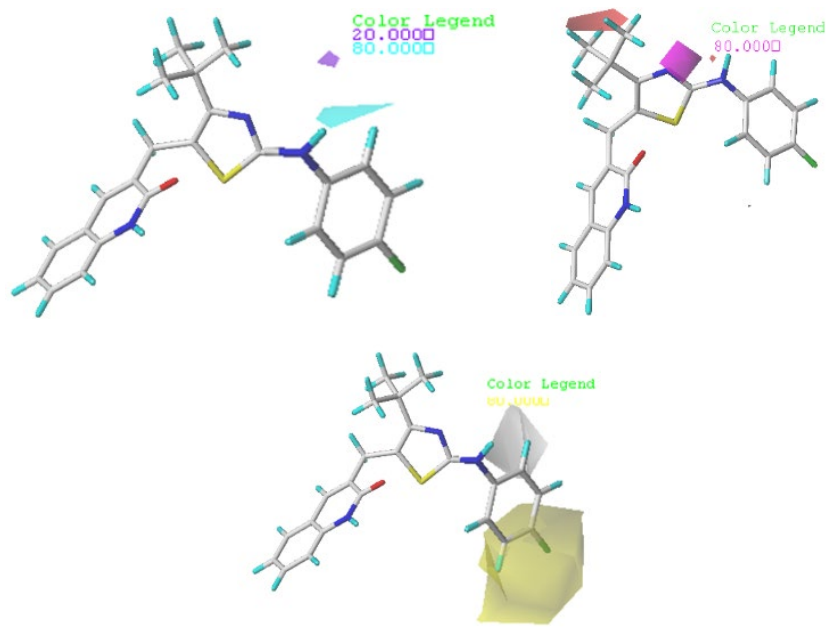

Fig. 5. Contour maps of CoMSIA analysis for compound 6. (a) H-bond donor field, (b) H-bond acceptor field, (c) Hydrophobic field.

\section{Y-randomization test}

Table 4 shows the results of nine random shuffles for the Y-randomization test. According to the Yrandomization test, the $\mathrm{Q}^{2}$ and $\mathrm{R}^{2}$ values obtained by the ten iterations were extremely lower than those of the original models. Consequently, the probability of an accidental correlation has been ruled out, indicating that the original models obtained are extremely trustworthy and accurate. 
Table 4. $\mathrm{Q}^{2}$ and $\mathrm{R}^{2}$ values of the $\mathrm{Y}$-randomization test.

\begin{tabular}{|c|c|c|c|c|}
\hline \multirow{2}{*}{ Iteration } & \multicolumn{2}{|c|}{ CoMFA } & \multicolumn{2}{c|}{ CoMSIA } \\
\cline { 2 - 5 } & $\mathbf{Q}^{\mathbf{2}}$ & $\mathbf{R}^{\mathbf{2}}$ & $\mathbf{Q}^{\mathbf{2}}$ & $\mathbf{R}^{\mathbf{2}}$ \\
\hline $\mathbf{1}$ & 0.102 & 0.366 & 0.099 & 0.351 \\
\hline $\mathbf{2}$ & 0.009 & 0.124 & 0.002 & 0.109 \\
\hline $\mathbf{3}$ & -0.108 & 0.082 & -0.104 & 0.078 \\
\hline $\mathbf{4}$ & 0.071 & 0.189 & 0.068 & 0.185 \\
\hline $\mathbf{5}$ & -0.044 & 0.121 & -0.065 & 0.110 \\
\hline $\mathbf{6}$ & 0.125 & 0.404 & 0.117 & 0.376 \\
\hline $\mathbf{7}$ & 0.184 & 0.296 & 0.147 & 0.246 \\
\hline $\mathbf{8}$ & 0.121 & 0.301 & 0.101 & 0.289 \\
\hline $\mathbf{9}$ & -0.112 & -0.275 & -0.129 & -0.281 \\
\hline
\end{tabular}

\section{Design and prediction}

The aim of this research is to develop new tubulin inhibitors. Few structural features obtained from contour maps are incorporated into the tetrahydroquinoline scaffold in the form of R1 and R2 substitutions. According to the significant structure information obtained, we determined the modified regions of this class of compounds. Considering the yellow hydrophobic contour map around the $\mathrm{C}-4$ position, the phenyl ring of the molecular skeleton played a worthy role in hydrophobic contacts. The A R1 region including the white contour is located near the $\mathrm{C}-2$ position of the aminothiazole phenyl ring, demonstrating that the presence of a hydrophilic substituent contributes to the enhancing activity. Electronegative groups substituted in the C-4 position of the R1 region would be beneficial for the improvement of the inhibitory activity, while electropositive groups substituted in the remaining position of R1 may increase the activity. The butyl-linked thiazole core of R2 was highlighted in green and red, indicating that adding HBD groups with less volume at this position may enhance the inhibitory activity. Consequently, four 2-oxoquinoline arylaminothiazoles (D1D4) targeting tubulin were designed with an increased potency profile. The predicted $\mathrm{pIC}_{50}$ values of the four new compounds are significantly greater than that of compound 6, indicating that the developed QSAR models were found to be useful in the design of new and successful compounds. The structures of the newly designed molecules and their predicted $\mathrm{pIC}_{50}$ and docking scores are given in Table 5.

Table 5. New designed molecules and their predicted $\mathrm{pIC}_{50}$ and docking score

\begin{tabular}{|c|c|c|c|c|c|}
\hline \multirow{2}{*}{$\mathbf{N}^{\circ}$} & \multirow{2}{*}{$\mathbf{R}_{\mathbf{1}}$} & \multirow{2}{*}{$\mathbf{R}_{2}$} & \multicolumn{2}{|c|}{ Predicted pIC 50} & \multirow{2}{*}{$\begin{array}{c}\text { Total score } \\
\text { Surflex Dock }\end{array}$} \\
\hline & & & CoMFA & CoMSIA & \\
\hline \multicolumn{6}{|c|}{ The most active compound } \\
\hline Comp 6 & $4-\mathrm{F}$ & Tert-Butyl & 5.356 & 5.377 & 3.8095 \\
\hline \multicolumn{6}{|c|}{ The newly designed compound } \\
\hline D1 & $2-\mathrm{NH}_{2}-4-\mathrm{CF}_{3}$ & Butyl & 5.696 & 5.677 & 6.1905 \\
\hline D2 & $2-\mathrm{OH}-4-\mathrm{CF}_{3}$ & Butyl & 5.675 & 5.683 & 5.9665 \\
\hline D3 & $2-\mathrm{F}-4-\mathrm{CF}_{3}$ & Butyl & 5.587 & 5.599 & 5.6116 \\
\hline D4 & $2-\mathrm{NH}_{2}-4-\mathrm{CN}$ & Butyl & 5.407 & 5.437 & 5.3914 \\
\hline
\end{tabular}




\section{Molecular docking}

Following QSAR analysis, we conducted molecular docking to clarify the differences of the activity between bioactive molecules, to figure out which binding mechanisms would support the ligand-protein interaction, and to generate insights for the design of bioactive molecules in the future. Before performing the docking study, the co-crystal ligand (colchicine) was redocked into the binding pocket to verify the reliability of the docking method. As shown in Fig. 6, the redocked conformation of 3E22 was highly consistent with its co-crystallized ligand conformation and the RMSD of the two conformations was $0.326 \AA(<2.0 \AA)$. This indicated that the performed docking protocol was reliable and could be used for subsequent study.

Docking results are listed in Table 5. The docking outcome was expressed in the Surflex-Dock as a Total Score, which calculates ligand binding affinity based on contributions from various interactions. The total score of the four newly designed compounds was investigated as judgment criteria. Newly designed compounds had a higher Total Score as compared to compound $\mathbf{6}$, indicating a greater affinity with the target protein. The newly designed compounds D1, D2, D3 and D4 and compound 6 were selected for further binding mode analysis by comparing their interactions with tubulin protein. The explanation for the difference in potency might be fairly explained in a reasonable way, implying that the binding mode of small molecules could be the real binding mode.

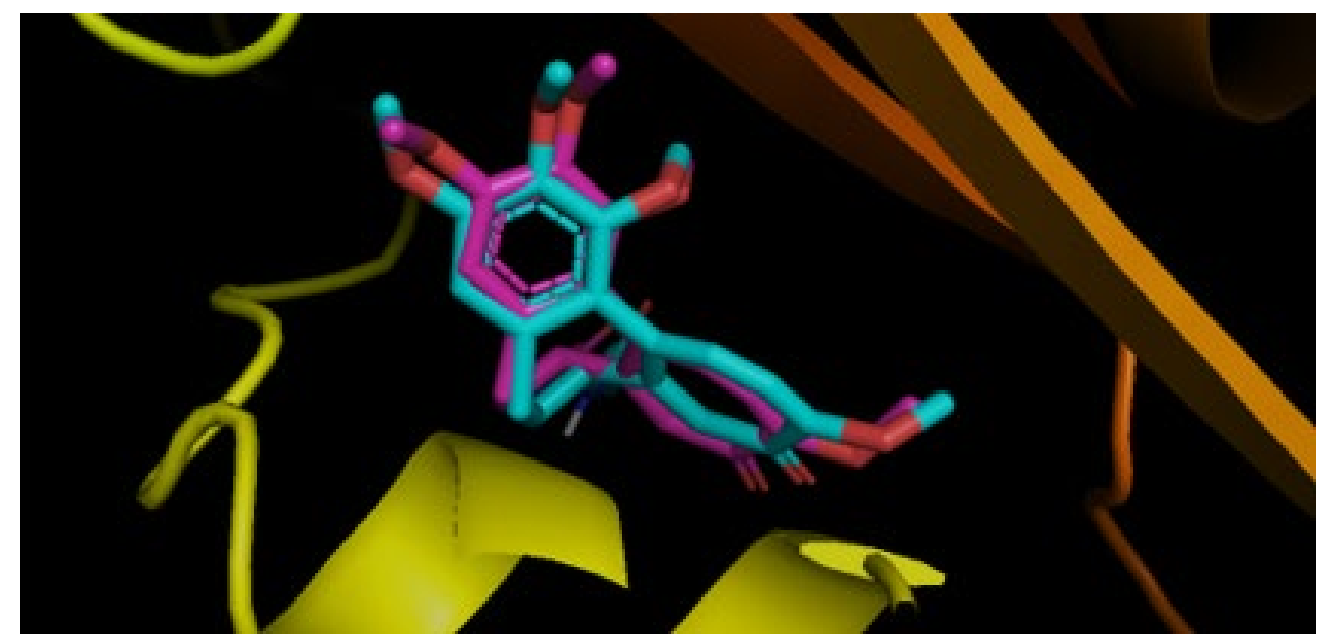

Fig. 6. Superimposition of the redocking pose (magenta) and the ligand pose in the co-crystal structure (cyan).

\section{Interactive mode of compound 6 in the active site of tubulin protein}

Among the selected docked compounds, compound $\mathbf{6}$ was recognized with the lowest total score of 3.8095. Fig. 7 depicts the active site of tubulin, where the fluorine group of the phenyl ring of compound 6 forms one hydrogen-bonding interaction with surrounding residue Asn 167. Another favorable pi-sigma interaction made up by Leu 248 was in contact with the quinoline moiety. Moreover, the alkyl chain on the thiazole group is buried in a hydrophobic cavity involving of residues Ala 316, Val 318 and Ile 378, and the phenyl ring forms several electrostatic interactions with the surrounding residues Leu 242, Val 238 and Leu 354, which can improve the potency. 


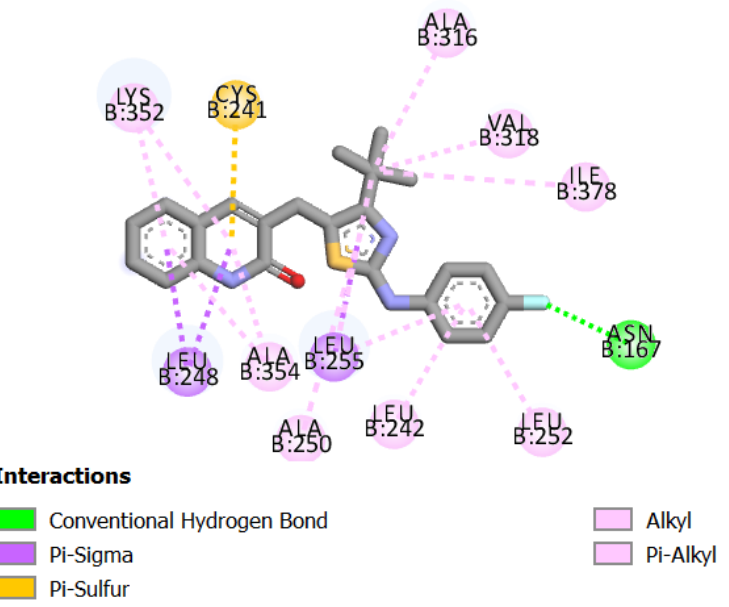

Fig. 7. The most active compound 6 binding into tubulin.

\section{Interactive mode of compound D1 in the active site of tubulin protein}

The newly designed compound D1 was acknowledged with a maximum total score of 6.1905. The hetrifluoromethyl group of the phenyl ring on the upper right side forms a hydrogen bond with the backbone amino acid Gln 136, as shown in Fig. 8(a). In particular, two strong hydrogen-bonding interactions were also formed between the residues of Val 238 and Thr 239 and the primary amino group of the same phenyl. Hydrophobic interactions were observed between the alkyl chain of the thiazole group with residues of Val 318 and Ala 316. In addition, the 2-oxoquinoline scaffold formed an -alkyl effect with the residues Ala 316 and Lys 352, while the same effect was observed for the phenyl ring with Leu 252 and Leu 242. Also, Met 259 had an -sulfur interaction with the quinoline group of the compound D1. Noticeably, the observed interactions are stabilizing interactions and beneficial for potency.

\section{Interactive mode of compound $\mathrm{D} 2$ in the active site of tubulin protein}

As depicted in Fig. 8(b), docking results show that compound D2 is located deep in the tubulin cavity enclosed by hydrogen bond residues. Obviously, the hydroxyl group in the second position of the phenyl ring extends into a pocket bordered by residues Thr 239 and Val 238. The primary amino group on the core forms a strong hydrogen bonding interaction with the surrounding residue Lys 254. Two strong hydrogen-bonding interactions were also formed between the residues of Gln 136 and Asn 167. Consequently, the abovementioned groups provide significant hydrogen bonding interactions for tubulin inhibitory binding. Furthermore, the alkyl chain of the thiazole group is located against the active pocket, resulting in three hydrophobic interactions with Val 318, Ala 316, and Ala 354. The compound D2 binds to a wide pocket containing several hydrophobic interactions with residues Tyr 202, Leu 242, Leu 248, Leu 255 and Ala 250.

\section{Interactive mode of compound D3 in the active site of tubulin protein}

As shown in Fig.8(c), the primary amino group connecting the thiazole ring and the phenyl moiety of the compound D3 forms a hydrogen bonding interaction with Lys 254. Another favorable interaction of hydrogen bonding was formed by the nitrogen atom of the 2-oxoquinoline scaffold with the surrounding residue of Val 238. The fluorine atoms of trifluoromethyl on the phenyl moiety are surrounded by Asn 350, Val 351, and Asn 350, while only Ile1083 and Gln114 are observed the fluorine located at the second position of the same phenyl. The alkyl chain in the thiazole group is mainly situated in the hydrophobic pocket formed by residues Leu 242, Leu 252, Leu 248 and Ala 250. Residues of Met 259, Lys 352, Cys 241 and Ala 316 were observed to form further hydrophobic interactions with the newly designed compound D3. This may be another explanation for the stronger activity of compound D3. 


\section{Interactive mode of compound D4 in the active site of tubulin protein}

The binding mode of compound $\mathbf{D} 4$ reveals that it bonds inside the binding pocket with a total score of 5.3914. It is clear that the oxygen atom of the quinoline moiety is surrounded by hydrogen bonding residues (Asn 249 and Ala 250), as depicted in Fig. 8(d). The same oxygen atom resides in the active pocket, forming a carbon hydrogen bond with Lys 254. A pi-lone pair interaction exists between Val 238 and the phenyl ring containing the modified substituents. It is also worth mentioning that the compound D4 also forms several hydrophobic interactions.
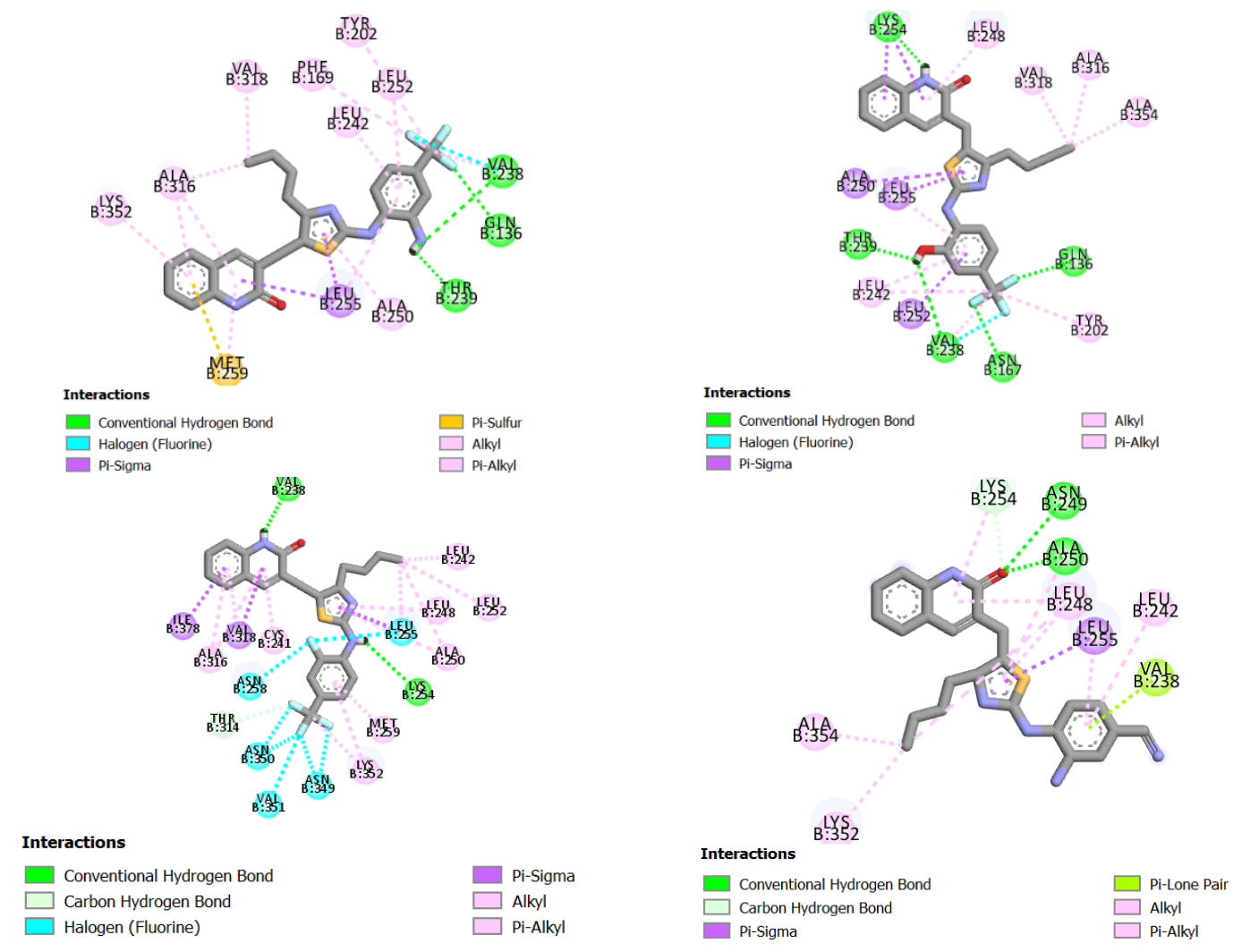

Fig. 8. The newly designed compounds binding into tubulin. (a): Compound D1, (b): Compound D2, (c): Compound D3, (d): Compound D4.

\section{Molecular dynamics simulation}

The $50 \mathrm{~ns}$ MD simulation was performed with 3E22-colchicine in complex with tubulin and two selected tubulin-compound complexes (Compound D1 and Compound 6) to examine the stability of proteinligand interactions. Fig. 9 represents the plot of RMSF of compound D1 backbone atoms during 50ns simulation times, while Fig. 10 shows the plots of RMSD, Rg, and SASA of the protein-compound D1 complex. Fig. 11 depicts the plot of RMSF of compound 6 backbone atoms during 50ns simulation times, while Fig. 12 shows the plots of RMSD, Rg, and SASA of the protein-compound 6 complex.

To characterize the flexibility of residues present in the protein during $50 \mathrm{~ns}$, RMSF values were also calculated. As shown in Fig. 9, a low RMSF is detected for most of the residues during the interaction of the Compound D1 bound complex, signifying that the protein has good dynamic stability. Whereas slightly nonsignificant fluctuations were observed for the binding of Compound $\mathbf{6}$ to the target protein as depicted in Fig. 
11. The highest degree of flexibility is detected for compound D1, since less fluctuation means greater protein structural stability.

Fig. 10 displays the RMSD of the heavy atoms of tubulin over $50 \mathrm{~ns}$. The RMSD of the compound D1 bound complex levels off at 1.62 after $15 \mathrm{~ns}$, indicating that the docking complex has entered a metastable state, and then an equilibrated system is obtained in complex D1 for the rest of the time. The curve of the Compound 6 bound complex went up slightly with much more deviation in the RMSD plot, and then turned into a sustained period of stability. RMSD analysis indicates that the designed molecule D1 forms a stable complex with the protein throughout simulations. The radius of gyration $(\mathrm{Rg})$ was also applied to represent the changes in the compactness of the protein structure throughout the simulation time (Figures 10 and 12). It is revealed from the Rg plot that the Rg values of Compound D1 bound complex varied in a small range of 4.95 and 5.40 for 50ns. However, the values basically stabilized in the same range after $11 \mathrm{~ns}$ until the end of the simulation. The $\mathrm{Rg}$ plot shows that there is some change in the compactness of the folding of tubulin after binding of compound $\mathbf{6}$. The Rg results indicate that the ductility of the protein was good for compound D1 throughout simulation time. Similarly, the SASA analysis was explored to determine the solvent accessibility of the complex (Figures 10 and 12). The SASA of the protein-Compound D1 complex fluctuated between 190 and 675 during the MD simulations and the average value of SASA was found to be 16342. Furthermore, the SASA plot revealed that the structure of Compound $\mathbf{6}$ was relatively instable during the simulation time.

Overall, the RMSF, RMSD, Rg and SASA results, strongly support the docking findings, suggesting that Compound D1 could be the most effective ligand for modulating tubulin activity.

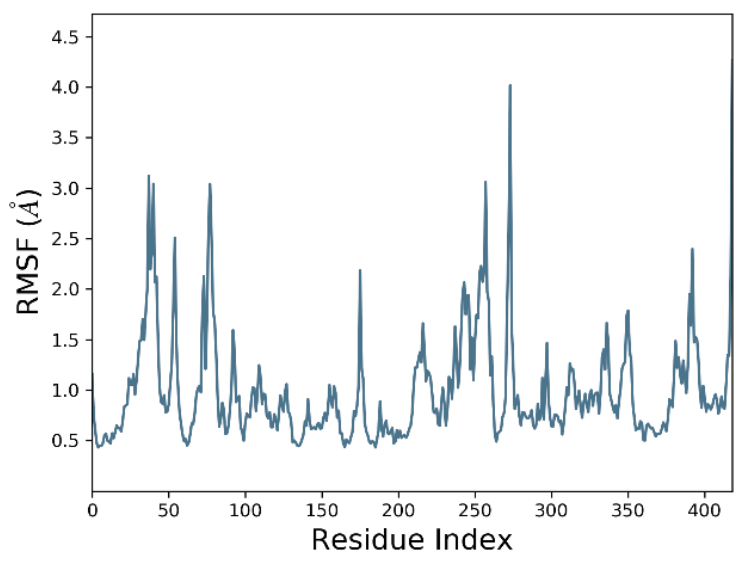

Fig. 9. The RMSF of compound D1 backbone atoms during 50ns simulation times

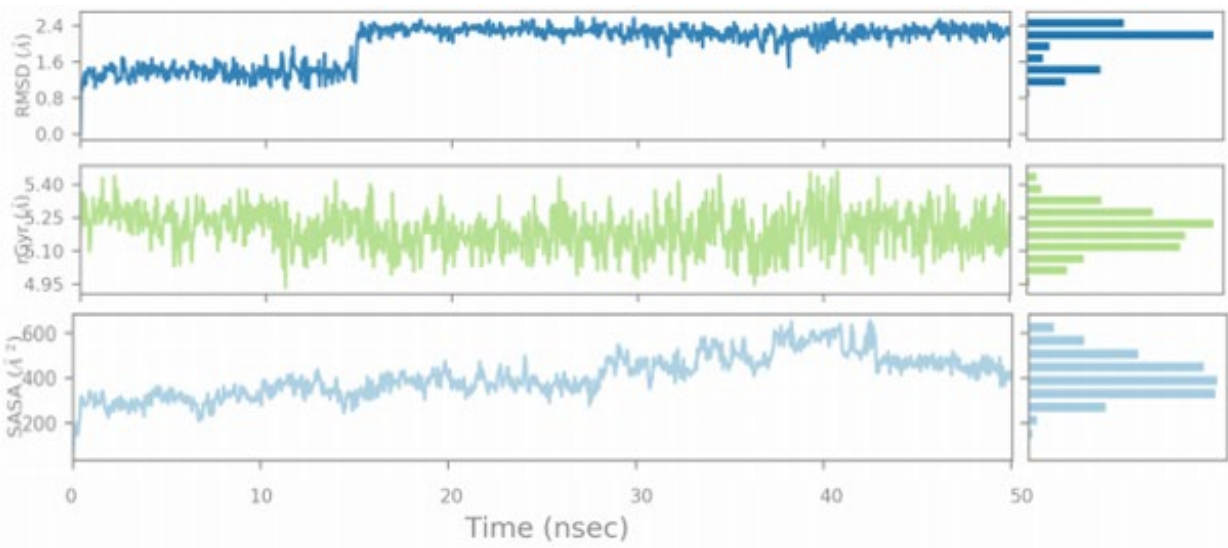

Fig. 10. The RMSD, Rg and SASA of protein-compound D1 complex in MD simulation. 


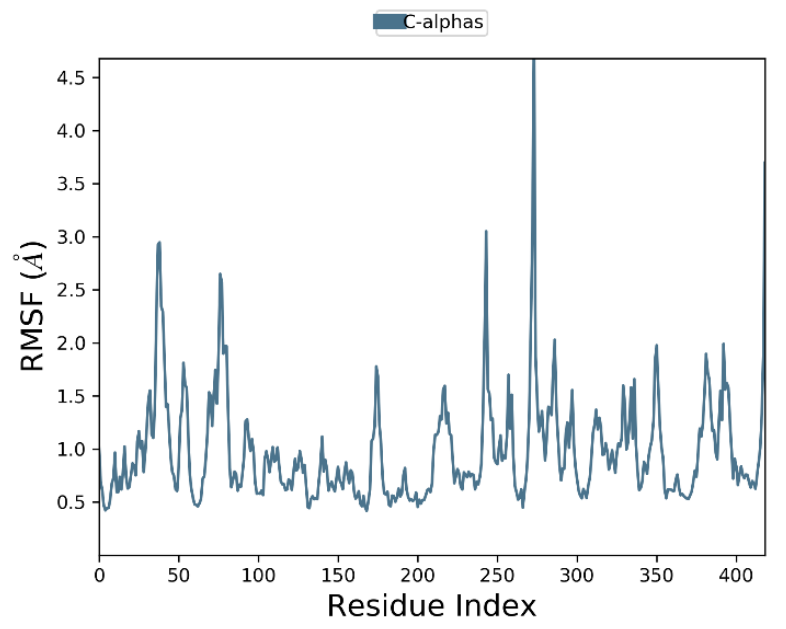

Fig. 11. The RMSF of compound 6 backbone atoms during 50ns simulation times.

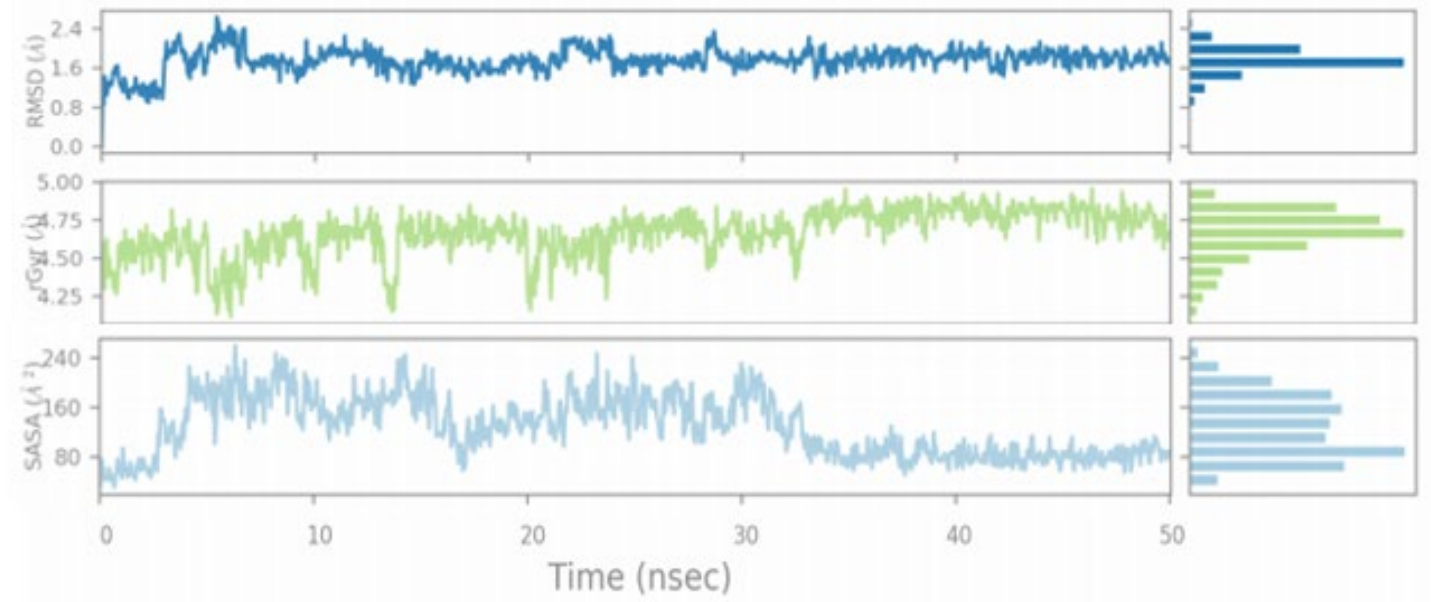

Fig. 12. The RMSD, Rg and SASA of protein-compound 6 complex in 50ns MD simulation.

\section{Conclusion}

For the objective of developing 3D-QSAR models based on Ligand-guided CoMFA and CoMSIA methods, a dataset of 25 2-oxoquinoline arylaminothiazole derivatives as potent tubulin inhibitors with cytotoxicity activity against the HeLa cancer cell line. The findings of two accurate CoMFA and CoMSIA models were used to clarify the relationship between the reported derivatives and their inhibition mechanisms. Consequently, four novel tubulin inhibitors (D1, D2, D3, and D4) were designed based on the important information unveiled by the contour maps. The four new inhibitors and compound 6 were chosen for further binding mode analysis by comparing their interactions with tubulin protein, indicating that all designed compounds, especially compound D1, remained stable inside the identified protein. Finally, two ligand-protein complexes (compound D1 and compound 6) were subjected to $50 \mathrm{~ns}$ MD simulation, and D1 verified the accuracy of the docking findings, indicating that our research has effectively revealed the binding mechanism of tubulin inhibition, which may be useful in the development of new selective tubulin inhibitors. 
Therefore, it must be checked whether this type of sterilization does not produce changes in its physicochemical properties or does not cause a significant loss of content, which affect the therapeutic effect. Based on the obtained results, it was ascertained that MS-MS spectroscopy technique is appropriate to be employed as an important method in monitoring the radiosterilization of tryptophan isomers and/or pharmaceutical delivering systems containing tryptophan as main ingredient.

The changes detected by DSC correspond to the differences indicated by HPLC i.e., a small mass loss of the active substance, which means that tryptophan isomers are resistant to ionising radiation (radiochemical stability) thus being safely subjected to sterilisation by irradiation.

\section{Acknowledgments}

We dedicated this work to the "Moroccan Association of Chemists Theorists" (MACT) for its pertinent help concerning the programs.

\section{References}

1. Siegel, R. L.; Miller, K. D.; Jemal, A. CA. Cancer J. Clin. 2019, 69, 7-34. DOI: https://doi.org/10.3322/caac.21551

2. Sana, S.; Tokala, R.; Bajaj, D. M.; Nagesh, N.; Bokara, K. K.; Kiranmai, G; Lakshmi, U. J.; Vadlamani, S.; Talla, V. Shankaraiah, N. Bioorg. Chem. 2019, 93, 103317. DOI: https://doi.org/10.1016/j.bioorg.2019.103317

3. Kaur, R.; Kaur, G.; Gill, R. K.; Soni, R.; Bariwal, J. Eur. J. Med. Chem. 2014, 87, 89-124. DOI: https://doi.org/10.1016/j.ejmech.2014.09.051

4. Liu, Y.-N.; Wang, J.-J.; Ji, Y.-T.; Zhao, G.-D.; Tang, L.-Q.; Zhang, C.-M.; Guo, X.-L.; Liu, Z.-P. J. Med. Chem. 2016, 59, 5341-5355. DOI: https://doi.org/10.1021/acs.jmedchem.6b00071

5. Stanton, R. A.; Gernert, K. M.; Nettles, J. H.; Aneja, R. Med. Res. Rev. 2011, 31, 443-481. https://doi.org/10.1002/med.20242

6. Mukhtar, E.; Adhami, V. M.; Mukhtar, H. Mol. Cancer Ther. 2014, 13, 275-284. DOI: https://doi.org/10.1158/1535-7163.MCT-13-0791

7. Bates, D.; Eastman, A. Br. J. Clin. Pharmacol. 2017, 83, 255-268. DOI: https://doi.org/10.1111/bcp.13126

8. Batran, R. Z.; Kassem, A. F.; Abbas, E. M. H.; Elseginy, S. A.; Mounier, M. M. Med. Chem. 2018, 26, 3474-3490. DOI: https://doi.org/10.1016/j.bmc.2018.05.022

9. Vuuren, R. J.; Visagie, M. H.; Theron, A. E.; Joubert, A. M. Cancer Chemother Pharmacol. 2015, 76, 1101-1112. https://doi.org/10.1007/s00280-015-2903-8

10. Jordan, M. A. Curr. Med. Chem. 2002, 2, 1-17. DOI: https://doi.org/10.2174/1568011023354290

11. Ibrahim, T. S.; Hawwas, M. M.; Malebari, A. M.; Taher, E. S.; Omar, A. M.; O’Boyle, N. M.; McLoughlin, E.; Abdel-Samii, Z. K. P. Pharmaceuticals. 2020, 13, 393. DOI: https://doi.org/10.3390/ph13110393

12. El-Naggar, A. M.; Eissa, I. H.; Belal, A.; EL-Sayed, A. A. RSC $A d v$. 2020, 10, 2791-2811. DOI: https://doi.org/10.1039/c9ra10094f

13. Thiyagamurthy, P.; Teja, C.; Naresh, K.; Gomathi, K.; Khan, F-R. N. Med. Chem. Res. 2020, 29, $1882-$ 1901. DOI: https://doi.org/10.1007/s00044-020-02606-4 
14. Kaur, R.; Kumar, K. S. Eur. J. Med. Chem. 2021, 215, 113220. DOI: https://doi.org/10.1016/j.ejmech.2021.113220

15. Mahajan, P.; Nikam, M.; Asrondkar, A.; Bobade, A.; Gill, C. J. Heterocyclic Chem. 2016, 000, 8. DOI: https://doi.org/10.1002/jhet.2722

16. Uddin, A.; Chawla, M.; Irfan, I.; Mahajan, S.; Singh, S.; Abid, M. RSC Med. Chem. 2021, 12, 24-42. DOI: https://doi.org/10.1039/d0md00244e

17. Katariya, K. D.; Shah, S. R.; Reddy, D. Bioorg. Chem. 2020, 94, 103406. DOI: https://doi.org/10.1016/j.bioorg.2019.103406

18. Fang, Y.; Wu, Z.; Xiao, M.; Li Wei, L.; Li, K.; Tang, Y.; Ye, J.; Xiang, J.; Hu, A. Bioorg. Chem. 2021, 106, 104469. DOI: https://doi.org/10.1016/j.bioorg.2020.104469

19. S TRIPOS Associates, Inc. Sybyl-X Molecular Modeling Software Packages., Version X-2.0. 2012

20. Clark, M.; Cramer, R. D.; Van Opdenbosch, N. J. Comput. Chem. 1989, 10, 982-1012. DOI: https://doi.org/10.1002/jcc.540100804

21. Purcell, W. P.; Singer, J. A. J. Chem. Eng. Data. 1967, 12, 235-246. DOI: https://doi.org/10.1021/je60033a020

22. El Khatabi, K.; Aanouz, I.; El-mernissi, R.; Khaldan, A.; Ajana, M. A.; Bouachrine, M.; Lakhlifi, T. Orbital Electron. J. Chem. 2020, 12, 172-181. DOI: https://doi.org/10.17807/orbital.v12i4.1467

23. Cramer, R. D.; Patterson, D. E.; Bunce, J. D. J. Am. Chem. Soc. 1988, 110, 5959-5967. DOI: https://doi.org/10.1021/ja00226a005

24. Klebe, G.; Abraham, U.; Mietzner, T. J. Med. Chem. 1994, 37, 4130-4146. DOI: https://doi.org/10.1021/jm00050a010

25. Ståhle, L.; Wold, S. Prog. Med. Chem. 1988, 25, 291-338. DOI: https://doi.org/10.1016/S0079$\underline{6468(08) 70281-9}$

26. EL-Mernissi, R.; El Khatabi, K.; Khaldan, A.; Ajana, M. A.; Bouachrine, M.; Lakhlifi, T. J. Mater. Environ. Sci. 2020, 11, 952-962.

27. Golbraikh, A.; Tropsha, A. J. Mol. Graph. Model. 2002, 20, 269-276. DOI: https://doi.org/10.1016/S1093-3263(01)00123-1

28. Tropsha, A.; Gramatica, P.; Gombar, V. QSAR Comb. Sci. 2003, 22, 69-77. DOI: https://doi.org/10.1002/qsar.200390007

29. Baroni, M.; Clementi, S.; Cruciani, G.; Costantino, G.; Riganelli, D.; Oberrauch, E. J. Chemom. 1992, 6, 347-356. DOI: https://doi.org/10.1002/cem.1180060605

30. Rücker, C.; Rücker, G.; Meringer, M. J. Chem. Inf. Model. 2007, 47, 2345-2357. DOI: https://doi.org/10.1021/ci700157b

31. Discovery Studio Predictive Science Application | Dassault Systèmes BIOVIA https://www.3dsbiovia.com/products/collaborative-science/biovia-discovery-studio/ (accessed Feb 6, 2020).

32. DeLano, W. L. DeLano. PyMOL Mol. Graph. Syst. DeLano Sci. San Carlos CA USA 2002.

33. Kaminski, G. A.; Friesner, R. A.; Tirado-Rives, J.; Jorgensen, W. L. J. Phys. Chem. B. 2001, 105, 6474-6487. DOI: https://doi.org/10.1021/jp003919d

34. Schrödinger Release 2020-1: Desmond Molecular Dynamics System, D. E. Shaw Research, New York, NY, 2020. Maestro-Desmond Interoperability Tools, Schrödinger, New York, NY, 2020.

35. Nam, K.; Gao, J.; York, D. M. J. Chem. Theory Comput. 2005, 1, 2-13. DOI: https://doi.org/10.1021/ct049941i 